\title{
Selective Nerve Root Blocks: A New Technique Using Electrical STIMULATION
}

\begin{abstract}
Robert F. Haynsworth Jr, MD
Selective nerve root blocks (SNRB) have been used for many years as a diagnostic tool in patients with low back pain with radicular symptoms. However the accuracy, specificity, and sensitivity of these blocks has been questioned as a screening tool for spine surgery. The utility of current SNRB techniques relies primarily on the relief of pain when local anesthetic is injected. However, patient responses are often non specific, and pain relief after in-

jecting local anesthetic is often difficult to interpret. A new technique for performing SNRB using electrical stimulation is described in this article. The technique has been developed in order to reproduce radicular pain by stimulation with electrical current rather than to rely on a response to local anesthetic injection. The technique decreases the reliance on spread of local anesthetic for interpretation, and can therefore reduce false positive results from

too much anesthetic (epidural spread affecting more than one nerve root) or not enough anesthetic (block peripheral to the area of inflammation or the "pain generator"). By stimulating several nerve roots in random order in a blind fashion to the patient, the technique can also eliminate placebo responders.

Keywords: selective nerve root block, transforaminal nerve block, anesthetic block nerve root, radicular pain.
\end{abstract}

Selective nerve root block (SNRB) has been used as a diagnostic and therapeutic tool in patients with radicular pain for many years (1-6). However the accuracy of these blocks in identifying irritated nerve roots has been questioned $(7,8)$. The utility of current SNRB techniques rely on reproduction of pain by mechanical stimulation with the needle tip, as well interpretation of pain relief after local anesthetic injection $(9,10)$. Several problems make current techniques of SNRB difficult to perform as well as hard to interpret. Success with current methods of SNRB depends heavily on the experience of the provider. Provocation of a nerve root with a needle causes a quick sharp "jab" which is often too short a stimulus for the patient to interpret, and can result in damage to the nerve root if done repeatedly. Other structures near the spine (ligaments, dorsal fascia, and intervertebral disc) have been reported to cause radicular pain when stimulated with a needle, which could lead to false positive results $(11,12)$.

From Baylor University Medical Center, Dallas, Texas. Address Correspondence: Robert F. Haynsworth Jr, MD, Baylor University Medical Center, 3600 Gaston Ave, Suite 360, Dallas, Texas-75246. E-mail: rhaynsworth@attbi.com

Funding: There was no financial interest between the author and any manufacturer or commercial product discussed in this article. No funding from a federal/state agency, nonprofit foundation, or commercial entity was used for this research in this article.
Pain relief after local anesthetic injection around a nerve root is also difficult to interpret. Pain relief should theoretically not occur unless the local anesthetic gets proximal to the pain generator (that is proximal to where the nerve root is being irritated by disc material, bone spurs, or scar tissue). However, North et al (13) showed that peripheral sciatic nerve blocks could relieve pain originating more centrally in the lumbosacral spine. Other investigators $(14,15)$ have shown that even central thalamic pain can be improved by injecting local anes-

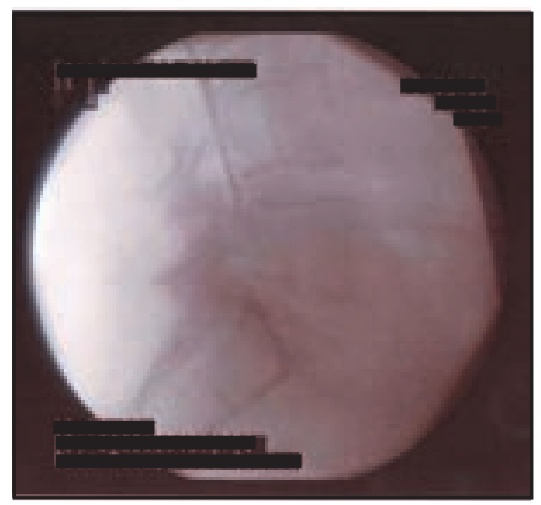

Fig. 1. Lateral radiograph showing needle placed at L5 nerve root. After injection of $1.2 \mathrm{~mL}$ of contrast, epidural spread can be seen extending up to L3 rather than remaining localized at the L5 area. thetic distally in the area where the pain is being perceived. The implication is that many patients are said to have a positive result after SNRB, when they are actually responding to a peripheral block. All these problems can decrease the accuracy rate of conventional blocks.

A new technique for performing SNRB using electrical stimulation is described in this

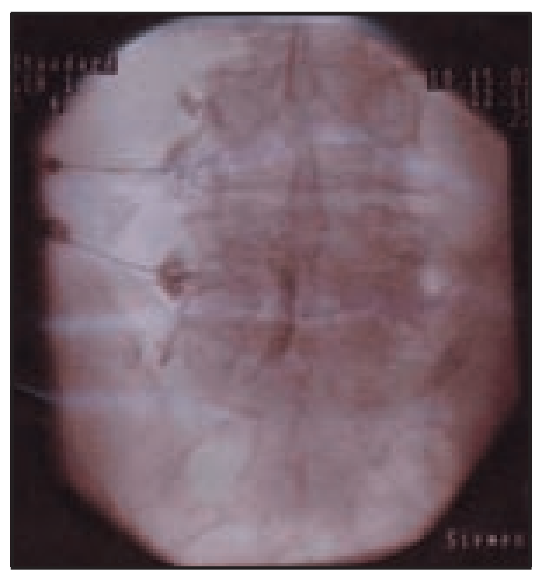

Fig. 2. AP radiograph in a patient with severe degenerative disc disease. The needle at L4 shows good outline of the L4 nerve root and no visible epidural spread. The needle at L5 shows spread along the L5 nerve root. However epidural spread to the S1 nerve root can also be seen from the L5 needle. Both needles were injected with $0.75 \mathrm{~mL}$ of contrast. 
article. The technique differs from conventional techniques by relying on stimulation with electrical current, rather than mechanical irritation or interpretation of local anesthetic spread. False positive results from too much anesthetic (epidural spread affecting more than one nerve root), or not enough anesthetic (block peripheral to the area of inflammation) can be reduced (Figs. 1 and 2). False positive responders can be decreased by the ability to stimulate multiple nerve roots repeatedly in a blinded fashion to the patient.

\section{Methods}

The patient is placed in the prone position on a fluoroscopy table. A pillow is placed under the pelvis if tolerated. Mild sedation is used if the patient is overly anxious. Needles should be guided into the foramina at the suspected level causing pain, as well as into the foramina above and below this level to allow the patient to compare adjacent nerve roots. Usual techniques for foraminal needle placement have been well described $(1,16)$. 22 G 3.5-inch insulated needles are preferred, while $22 \mathrm{G} 5$ or 7 -inch needles are used for large patients.

For final position in the lumbar area, needles should be slightly inside the posterior edge of the foramina according to lateral fluoroscopy, and at the lateral edge of the vertebral body on AP view. This is considered the safe zone for needle placement (16). For the sacral foramina, lateral view should show the needle to slightly deeper than the posterior sacrum, but not through the anterior foramina into the pelvis. Once the needles have been placed, 0.1 to 0.2 milliliter of contrast (Omnipaque) is injected until an outline of each nerve root is achieved (Fig. 3).

To apply stimulation to the needles, a stimulator with the ability to generate pulses of at least $50 \mathrm{~Hz}$ (to stimulate sensory fibers) and the ability to adjust voltage output is necessary. The stimulator on the RFG-3C+ radiofrequency lesion generator (Radionics) works well because the rate and the output current can be adjusted easily (Fig. 4). A Radionics needle kit is opened and the 10 centimeter RF probe is connected to the radiofrequency lesion generator. The rate is set to $50 \mathrm{~Hz}$ and the current output is initially set to 0 $\mathrm{mV}$. The stylet of the insulated needle is removed slightly $(2-3 \mathrm{~cm}$.) At this point, the probe is touched anywhere along the stylet (Fig. 5). It just needs to make metal to metal contact with the stylet in order to stimulate. Current output is slowly increased until the patient begins to feel sensation down the leg. The voltage level needed to produce this response is record-

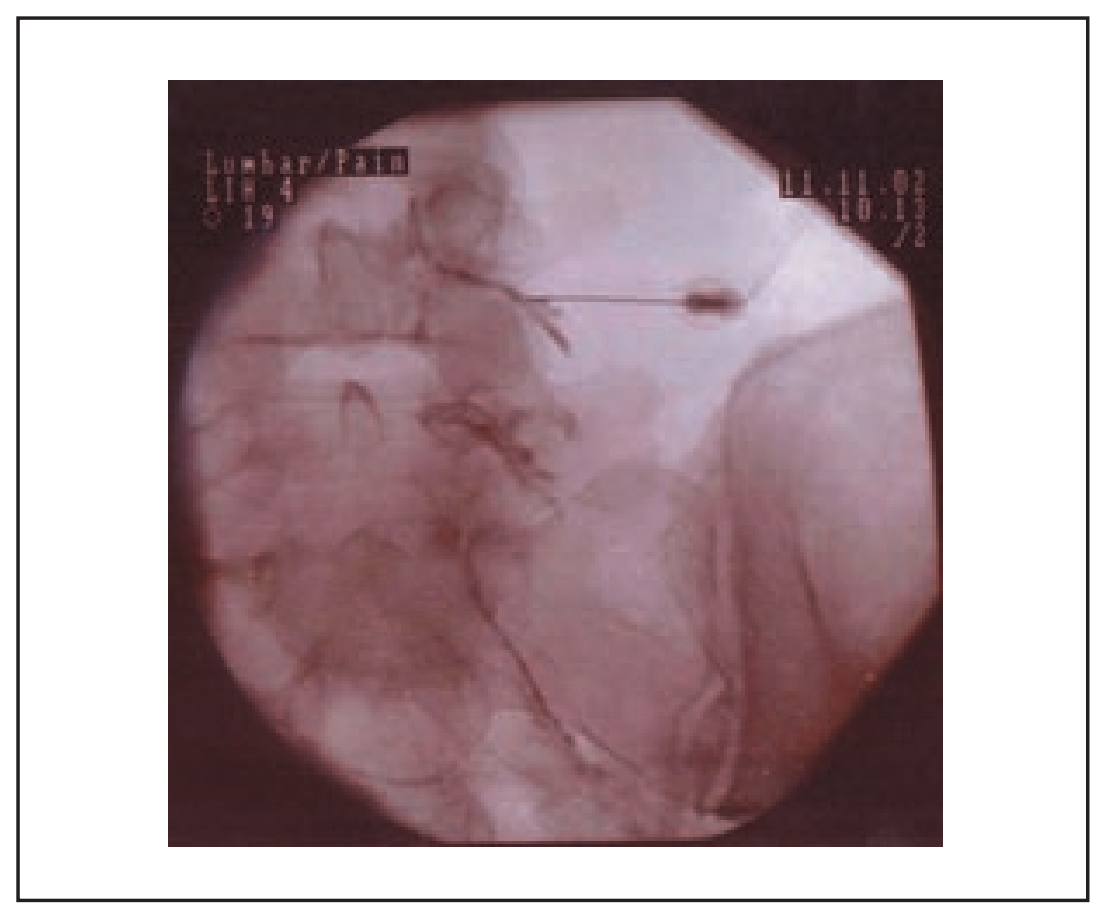

Fig. 3. AP radiograph showing contrast outlining the nerve roots of L4, L5, and $S 1$ on the right. ed. The patient is asked if this stimulation is in the same location of their usual pain. The current is then turned back to $0 \mathrm{mV}$, and the RF probe is moved to contact the next needle. Voltage is slowly increased again until the patient again feels stimulation. The voltage level for the second nerve root is recorded. The patient is asked again if this stimulation reproduces their usual pain, and how it differs from the first needle stimulation. The same procedure is carried out on the third nerve root. This process can be carried out multiple times to let the patient choose which needle most closely reproduces their radicular pain.

It should be noted that the current needed to produce stimulation will vary from needle to needle. This is because one needle may be closer to the nerve root than another. It is important that the stimulus be consistent between each nerve root. Therefore, the amount of current needed to first stimulate each nerve root needs to be used every time that particular needle is stimulated.

After the patient identifies which needle most closely reproduces their pain, stimulation is carried out again in reverse or random order. This serves as a control as the patient is blinded as to which nerve root is being stimulated.

Once the patient can consistently identify one nerve root as the one causing their pain, 0.1 to $0.2 \mathrm{~mL}$ of local anesthetic ( $2 \%$ lidocaine) is injected. Relief of pain should occur within several minutes. Once the pain resolves (indicating the nerve root is anesthetized) the nerve root is again stimulated with the same amount of current originally required to produce a response, and the patient should not feel any stimulation in their leg. The other nerve roots should also be stimulated (without injecting local anesthetic though them) to make sure they were not anesthetized. If the patient cannot feel stimulation through the non-injected needles, epidural spread of local anesthetic has occurred to the adjacent nerve roots. If the patient gets no relief of pain after injection of local anesthetic through the first needle, then anesthetic can be injected through the second needle. If no relief occurs, then the third needle should be injected.

Once the procedure is finished, then steroid (40mg methylprednisolone) can be injected through the needle which reproduced the symptoms. Results should 


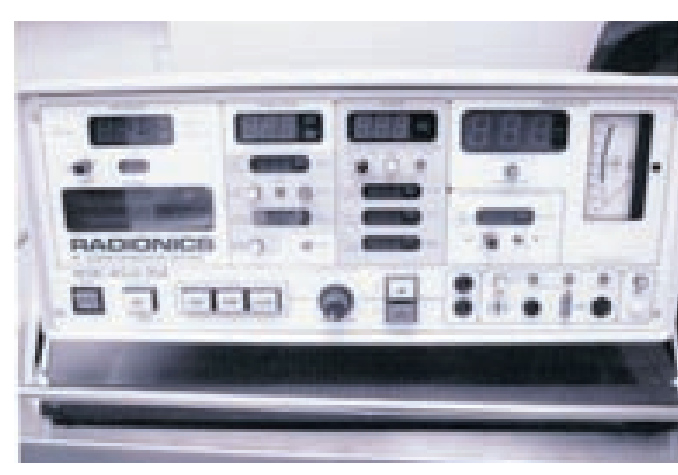

Fig. 4. Picture of the Radionics RFG-3C+ radiofrequency lesion generator which has a $50 \mathrm{~Hz}$ stimulator with adjustable voltage. (Radionics copyright 2001-2002 by Tyco Healthcare Group LP "Tyco").

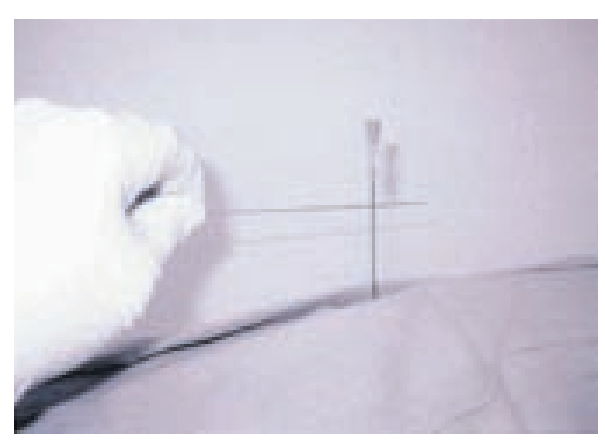

Fig. 5. Picture of Radionics RF $10 \mathrm{~cm}$ stimulating probe touching a foraminal needle. Rapid stimulation of several adjacent needles can be done be just touching the RF probe against the foraminal needle rather than placing the probe inside each needle as a stylet.

Table 1. Stimulated selective nerve root block results template

Patient:

Date:

Pre block pain (0-10):

$\begin{array}{lllllll}\text { Nerve root: } & \mathrm{L}_{3} & \mathrm{~L}_{4} & \mathrm{~L}_{5} & \mathrm{~S}_{1} & \text { Left } & \text { Right }\end{array}$

Stimulation voltage to reproduce pain: $\mathrm{mV}$.

Stimulation reproduces pain?

Not at all Somewhat Exactly

Post local anesthetic pain decrease: $\quad 0 \% \quad 10 \%$ $20 \% \quad 30 \% \quad 40 \% \quad 50 \%$

Yes (root not blocked) $60 \% \quad 70 \% \quad 80 \% \quad 90 \% \quad 100 \%$

Post anesthetic stimulation reproduces pain?

Yes (no epidural spread)

No (root blocked)

Able to stimulate non injected nerve roots?

$\begin{array}{lllllll}\text { Nerve root: } & \text { L3 } & \text { L4 } & \text { L5 } & \text { S1 } & \text { Left } & \text { Right }\end{array}$

Stimulation voltage to reproduce pain: $\mathrm{mV}$.

Stimulation reproduces pain?

Not at all Somewhat Exactly

Post local anesthetic pain decrease: $\quad 0 \% \quad 10 \%$

Post anesthetic stimulation reproduces pain?

$20 \% \quad 30 \% \quad 40 \% \quad 50 \%$

Yes (root not blocked)

$60 \% \quad 70 \% \quad 80 \%$

No (root blocked)

Able to stimulate non injected nerve roots?

Yes (no epidural spread)

No (epidural spread)

Stimulation voltage to reproduce pain: $\mathrm{mV}$.

Stimulation reproduces pain?

Post local anesthetic pain decrease: $\quad 0 \% \quad 10 \%$

Post anesthetic stimulation reproduces pain?

Able to stimulate non injected nerve roots?
Nerve root: $L$

L4

$\mathrm{L}_{5}$

S1

Left

Right

Not at all Somewhat

$20 \% \quad 30 \% \quad 40 \% \quad 50 \%$

Yes (root not blocked)

Yes (no epidural spread)
Exactly

$60 \% \quad 70 \% \quad 80 \% \quad 90 \% \quad 100 \%$

No (root blocked)

No (epidural spread)

Post procedure pain (0-10): 
be recorded on a template similar to the one in Table 1.

\section{Discussion}

The technique presented in this article has been developed to avoid problems inherent with current methods of selective nerve root block. Rather than anesthetizing or mechanically irritating the nerve root, stimulation provides a better method of identifying irritated nerve roots. Stimulating needles adjacent to the nerve root in question allows comparison which helps the patient to more precisely identify their pain. By changing the order of stimulation in a blinded fashion to the patient, false positives can be greatly reduced. False positive responses due to block of the nerve root distal to the source of irritation can also be reduced by using electrical stimulation rather local anesthetic (17).

Once nerve roots are identified consistently by stimulation, local anesthetic can then be injected for added confirmation. False positive responders due to epidural spread of anesthetic to adjacent nerve roots can be eliminated by re-stimulating non injected needles and confirming response at their original voltage.

\section{Conclusion}

In summary, preliminary results show the technique to be much more effective at not only identifying irritated nerve roots, but also more sensitive in identifying false responders. Instead of relying on pre- cise needle placement (as with the current methods for selective nerve root block), stimulated selective nerve root blocks allow placement anywhere along the nerve root. The technique can be a useful diagnostic as well as therapeutic tool in the patient with radicular symptoms.

\section{Author Affiliation:}

Robert F. Haynsworth Jr, MD

Clinical Director

Baylor University Medical Center

3600 Gaston Ave., Suite 360

Dallas, TX -745246

E-mail: rhaynsworth@attbi.com

\section{REFERENCES}

1. Adriani J. In Regional Anesthesia. Techniques and clinical application. $3^{\text {rd }}$ ed. WB Saunders, Philadelphia, 1967, p 261.

2. Dooley J, McBroom R, Taguchi $\mathrm{T}$ et al. Nerve root infiltration in the diagnosis of radicular pain. Spine 1988; 13:79-83.

3. Gamburd R. The use of selective injections in the lumbar spine. In Herring SA (ed). Physical Medicine and Rehabilitation Clinics of North America: Low Back Pain. WB Saunders, Philadelphia, 1991, pp 79-96.

4. Krempen J, Smith B. Nerve-root injection: A method for evaluation the etiology of sciatica. J Bone Joint Surg 1974; 56A: 1435-1444.

5. Manchikanti L. Transforaminal lumbar epidural steroid injections. Pain Physician 2000; 3:374-398.

6. Tajima T, Omata S, Kuramochi E et al. Selective lumbosacral radiculography and block. Orthop Surg 1976; 27:1053-1062.

7. Haueisen D, Smith B, Myers S et al. The diagnostic accuracy of spinal injection studies: their role in evaluation of recurrent sciatica. Clin Orthop 1985; 198:179-183.

8. Slosar P, White A, Wetzel T. The use of selective nerve root blocks: diagnostic, therapeutic, or placebo? Spine 1998; 23: 2253-2256.

9. Derby R, Kine G, Saal J et al. Response to steroid and duration of radicular pain as predictors of surgical outcome. Spine 1992; 17:176-183.

10. Takeshi T, Furukawa K, Kuramochi E. Selective lumbosacral radiculography and block. Spine 1980; 5:68-77.

11. Murphey F. Sources and patterns of pain in disc disease. Clin Neurosurg 1968; 15 : 343-351.

12. Steindler A, Luck J. Differential diagnosis of pain in the low back: Allocation of the source of pain by procaine hydrochloride method. JAMA 1938; 110:106-113.

13. North R, Kidd D, Zahurak M et al. Specificity of diagnostic nerve blocks: A prospective, randomized study of sciatica due to lumbosacral spine disease. Pain 1996; 65:77-85.

14. Kibler R, Nathan P. Relief of pain and paresthesiae by nerve block distal to a lesion. Neurol Neurosurg Psychiat 1960; 23:91-98.

15. Loh L, Nathan P, Schott G. Pain due to lesions of the central nervous system removed by sympathetic block. $\mathrm{Br}$ Med J 1981; 282:1026.

16. Derby R, Bogduk N, Kine G. Precision percutaneous blocking procedures for localizing spinal pain. Part 2. The lumbar neuraxial compartment. Pain Digest 1993; 3:175-188.

17. Kikuchi S, Hasue M, Nishiyama K et al. Anatomic and clinical studies of radicular symptoms. Spine 1984; 9:23-30. 